\title{
Marie-Anne Paveau, Georges-Élia Sarfati, Les Grandes théories de la linguistique. De la grammaire comparée à la pragmatique
}

\section{Anne Giaufret}

\section{(2) OpenEdition \\ Journals}

Édition électronique

URL : https://journals.openedition.org/studifrancesi/39942

DOI : 10.4000/studifrancesi.39942

ISSN : 2421-5856

Éditeur

Rosenberg \& Sellier

\section{Édition imprimée}

Date de publication : 1 décembre 2004

Pagination : 424-425

ISSN : 0039-2944

\section{Référence électronique}

Anne Giaufret, « Marie-Anne Paveau, Georges-Élia Sarfati, Les Grandes théories de la linguistique. De la grammaire comparée à la pragmatique », Studi Francesi [En ligne], 143 (XLVIII | II) | 2004, mis en ligne le 30 novembre 2015, consulté le 19 mai 2021. URL : http://journals.openedition.org/studifrancesi/ 39942 ; DOI : https://doi.org/10.4000/studifrancesi.39942

Ce document a été généré automatiquement le 19 mai 2021.

\section{cc) $(9)$}

Studi Francesi è distribuita con Licenza Creative Commons Attribuzione - Non commerciale - Non opere derivate 4.0 Internazionale. 


\title{
Marie-Anne Paveau, Georges-Élia Sarfati, Les Grandes théories de la linguistique. De la grammaire comparée à la pragmatique
}

\author{
Anne Giaufret
}

\section{RÉFÉRENCE}

MARIE-ANNE PAVEAU, GEORGES-ÉLIA SARFATI, Les Grandes théories de la linguistique. De la grammaire comparée à la pragmatique, s.l., Armand Colin/VUEF, 2003, pp. 256.

1 Le but de ce nouvel ouvrage sur l'histoire des théories linguistiques est de «donner une vision cohérente des conceptions linguistiques du XIXe et du XXe siècles», en répondant à une triple exigence: pédagogique, historique et épistémologique. Le volume, divisé en onze chapitres, se développe selon une "progression historique soucieuse de restituer les filiations»: les trois premiers chapitres présentent la linguistique pré-saussurienne (de la grammaire comparée, à la réaction néogrammairienne, au modèle de Meillet), alors qu'à partir du quatrième, consacrée à Saussure, sont présentées les grandes théories du XXe siècle, du structuralisme à la pragmatique, en passant par le fonctionnalisme, le formalisme, l'analyse du discours, la linguistique textuelle et la sémantique.

2 Les auteurs, qui affirment dans l'introduction vouloir donner «une vision cohérente des conceptions linguistiques du XIXe siècle, afin d'éviter les «limites restrictives» imposées par le point de vue courant selon lequel la linguistique commencerait avec Saussure, consacrent donc le premier chapitre de leur ouvrage à la grammaire comparée, depuis ses origines, au XVIIe siècle, à ses systématisations les plus complètes (Schlegel, Grimm, Rask et Bopp), aux théories de Humboldt et de Schleicher; le second aux retombées linguistiques du positivisme, avec la critique de l'historicisme, 
l'affirmation du primat des lois phonétiques et la naissance d'une nouvelle mouvance, celle des néo-grammairiens, qui s'exprime notamment en dehors de la France dans les travaux de $\mathrm{H}$. Paul et de Whitney, mais dans laquelle se situent pleinement les premiers travaux de Saussure; le troisième à la réception française de ces théories (notamment par Michel Bréal, dont les intuitions vont s'avérer fondamentales pour la sémantique de l'énonciation), qui seront reprises et développées par la réflexion éminemment théorique de Victor Henry ainsi que par l'analyse sociale du langage de Meillet.

Quand on arrive au Cours de linguistique générale de Saussure, donc, celui-ci se présente en même temps comme une continuité et une rupture avec le passé, car un atout de cet ouvrage est justement de bien montrer que «les étiquettes en -isme aplatissent les réalités théoriques» (p. 110) et qu'il s'agit souvent «de courants imbriqués les uns dans les autres, liés par des rapports de filiations et d'opposition et par des choix théoriques complexes»(p. 110). Mais ce choix du refus de la simplification se fait parfois malheureusement au détriment d'une certaine clarté, surtout si l'on considère que le public visé est un public d'étudiants.

4 La bibliographie générale est complète en ce qui concerne les auteurs traités; à la fin de chaque chapitre une mini-bibliographie indique en outre quelques références aux théories abordées.

5 Quoiqu'il soit regrettable que l'index se limite aux noms propres et qu'il n'y ait donc pas la possibilité de faire une recherche thématique à partir d'un courant théorique ou des termes et notions des sciences du langage, le but des auteurs, à savoir de contribuer «ne serait-ce que modestement, à l'enseignement d'une linguistique qui s'interroge sur ses enjeux», est certainement atteint. 